\title{
Search for E(5) symmetry in nuclei: The Ru isotopes
}

\author{
A. Frank, ${ }^{1,2}$ C. E. Alonso, ${ }^{3}$ and J. M. Arias $^{3}$ \\ ${ }^{1}$ Instituto de Ciencias Nucleares, UNAM, A. P. 70-543, Circuito Exterior, C. U., 04510 México, D. F., México \\ ${ }^{2}$ Centro de Ciencias Físicas, UNAM, Apdo. Postal 139-B, Cuernavaca, Morelos, México \\ ${ }^{3}$ Departamento de Física Atómica, Molecular y Nuclear, Facultad de Física, Universidad de Sevilla, Apartado 1065, 41080 Sevilla, Spain
}

(Received 17 July 2001; published 30 November 2001)

\begin{abstract}
We carry out an interacting boson model study of the Ru isotopes using a U(5)-SO(6) transitional Hamiltonian with fixed parameters, where the variation is due only to the change in boson number $N$. Transitional behavior in ${ }^{104} \mathrm{Ru}$ is compared with recent predictions of an $\mathrm{E}(5)$ critical symmetry, including a modified version with an alternative $\beta$ dependence for the $T^{(\mathrm{E} 2)}$ operator.
\end{abstract}

DOI: 10.1103/PhysRevC.65.014301

PACS number(s): 21.60.Fw, 21.10.Re, 21.10.Ky, 27.60.+j

\section{INTRODUCTION}

The study of phase-transitional behavior in physical systems is a difficult endeavor, since under these conditions structural changes occur very rapidly. In atomic nuclei the situation is complicated by the finite particle nature of these many-body systems. Shape-phase transitions can be described in nuclei in the framework of both the collective model [1] and the interacting boson model (IBM) [2]. In the latter case, the usual procedure is to consider a combination of symmetries, such as $\mathrm{U}(5)$ and $\mathrm{SO}(6)$, or $\mathrm{U}(5)$ and $\mathrm{SU}(3)$, each of which corresponds to stable structures such as spherical, $\gamma$ unstable, or deformed, for the $\mathrm{U}(5), \mathrm{SO}(6)$, and $\mathrm{SU}(3)$ limits of the model, respectively. For certain parameter values the nucleus undergoes a rapid transition from one kind of structure to the other [3]. The dynamical symmetries themselves represent idealized cases where analytical solutions can be found and all observables computed in analytic form [2], but one of the most striking successes of this model was the discovery of nuclei that closely follow these predictions [4]. Although a vast majority of nuclei exhibit a mixture of symmetries and require numerical diagonalizations to compute their properties, the symmetries nevertheless constitute important benchmarks from which nuclear behavior can be gauged. In this context, critical (or transitional) behavior cannot be described within the IBM in analytic, closed form. In two recent papers, however, Iachello has proposed a simple, analytic treatment of nuclei undergoing transitions from spherical to $\gamma$-unstable shapes [5] [E(5)] or from a spherical to a deformed configuration [6] $[\mathrm{X}(5)]$. These analyses have the attractive feature of being essentially parameter-free. Surprisingly enough, examples where both situations seem to be closely approximated were subsequently found by Casten and Zamfir [7,8], thus raising the possibility of studying whether $\mathrm{E}(5)$ and $\mathrm{X}(5)$ characteristics are widespread in transitional nuclei. If that would be the case they could represent a helpful theoretical tool in nuclear structure physics, in a sense complementing the role played by the IBM dynamical symmetries. In this paper we analyze under this new point of view a calculation of the Ru isotopes, which was carried out with an IBM Hamiltonian with fixed parameters [9], which we combine with a coherent-state analysis [10], in order to test the $\mathrm{E}(5)$ predictions. In subsequent work we plan to analyze the spherical to deformed regions using the same procedure and compare them with the $\mathrm{X}(5)$ calculations [11].

The paper is organized as follows. In Sec. II the critical point E(5) approach is revised. Section III is devoted to present the IBM description of Ru isotopes and discuss corresponding potential energy surfaces in relation to the $\mathrm{E}(5)$ symmetry. Finally, in Sec. IV the conclusions of this work are presented.

\section{THE E(5) APPROACH}

We start our discussion by giving a brief description of the $\mathrm{E}(5)$ analysis.

The new approach of Iachello starts from the BohrMottelson Hamiltonian,

$$
\begin{aligned}
H= & -\frac{\hbar^{2}}{2 B}\left[\frac{1}{\beta^{4}} \frac{\partial}{\partial \beta} \beta^{4} \frac{\partial}{\partial \beta}+\frac{1}{\beta^{2} \sin 3 \gamma} \frac{\partial}{\partial \gamma} \sin 3 \gamma \frac{\partial}{\partial \gamma}\right. \\
& \left.-\frac{1}{4 \beta^{2}} \sum_{\kappa} \frac{L_{\kappa}^{2}}{\sin ^{2}\left(\gamma-\frac{1}{3} \pi \kappa\right)}\right]+V(\beta, \gamma),
\end{aligned}
$$

where the $L_{\kappa}, \kappa=1,2,3$ are the body-fixed components of the angular momentum $[1,5]$. For situations where the potential does not depend on $\gamma$, one can factorize the solutions of Eq. (1) as

$$
\psi\left(\beta, \gamma, \theta_{i}\right)=f_{\xi, \tau}(\beta) \Phi^{\tau \mu L M}\left(\gamma, \theta_{i}\right),
$$

where the $\Phi$ functions were determined in Refs. [12,13]. These functions are eigenstates of any quadrupole Hamiltonian that displays $\mathrm{O}(5) \supset \mathrm{O}(3)$ symmetry, i.e., for $V(\beta, \gamma)=V(\beta)$. The $f(\beta)$ functions depend on the selection of the $\beta$ potential. The best known case is that of a fivedimensional oscillator $V(\beta) \approx \beta^{2}$ that leads to the Laguerre polynomials $[1,12,13]$.

Since in a phase-transitional situation one expects to have a particularly flat potential in $\beta$ (in this case, for a vibrator to $\gamma$-unstable rotor transition there is no $\gamma$ dependence), in Ref. [5] a five-dimensional infinite well is suggested as a good approximation, i.e., 


$$
\begin{array}{ll}
V(\beta)=0, & \beta \leqslant \beta_{\omega}, \\
V(\beta)=\infty, & \beta>\beta_{\omega},
\end{array}
$$

in which case the wave functions in $\beta$ take the simple form

$$
f_{\xi, \tau}(\beta)=C_{\xi, \tau} \beta^{-3 / 2} J_{\tau+3 / 2}\left(\frac{x_{\xi, \tau}}{\beta_{\omega}} \beta\right),
$$

where $\tau=0,1,2, \ldots$ labels the $\mathrm{SO}(5)$ group, $\xi$ is an index that enumerates the successive zeroes of the Bessel functions, $x_{\xi, \tau}$ is the $\xi$ th zero for a given $\tau, C_{\xi \tau}$ is a normalization, and $\beta_{\omega}$ is the range of the square potential in Eq. (3). The full states (2) can then be denoted by

$$
|\xi \tau \mu L M\rangle,
$$

where $\mu$ is a label that distinguishes between repeated $L$ 's for a given value of $\tau$. The definition of this index together with the $L$ content for each $\tau$ can be found in Refs. [2,12]. Since the states (5) can be used to generate a fivedimensional basis for the Euclidean group in five dimensions, they have been labeled by $\mathrm{E}(5)$. Using these states, all energies can be calculated exactly up to a scale

$$
E_{\xi, \tau}=\frac{\hbar^{2}}{2 B} \frac{\left(x_{\xi, \tau}\right)^{2}}{\beta_{\omega}^{2}} .
$$

In addition, assuming a first order quadrupole operator in the collective variables,

$$
T_{\mu}^{(E 2)}=t \alpha_{2 \mu}=t \beta\left[D_{\mu, 0}^{(2)} \cos \gamma+\frac{1}{\sqrt{2}}\left(D_{\mu, 2}^{(2)}+D_{\mu,-2}^{(2)}\right) \sin \gamma\right],
$$

it is likewise possible to calculate all $B(E 2)$ values in a parameter independent way (up to the scale factor $t$ ). One should note, however, that the latter depend on the particular form of Eq. (7). In the context of the geometric model, the next order in the quadrupole coordinates is given by [12]

$$
T_{\mu}^{(E 2)}=t\left(\alpha_{2 \mu}+\frac{\chi}{2 \sqrt{7}}\left[\alpha_{2} \times \alpha_{2}\right]_{\mu}^{(2)}\right)=t \sum_{m} D_{\mu m}^{(2) *} \bar{Q}_{m}^{(2)},
$$

where

$$
\begin{gathered}
\bar{Q}_{0}^{(2)}=\beta \cos \gamma-\sqrt{\frac{1}{14}} \chi \beta^{2} \cos (2 \gamma), \\
\bar{Q}_{2}^{(2)}=\bar{Q}_{-2}^{(2)}=\frac{1}{\sqrt{2}}\left[\beta \sin \gamma+\sqrt{\frac{1}{14}} \chi \beta^{2} \sin (2 \gamma)\right], \\
\bar{Q}_{1}^{(2)}=\bar{Q}_{-1}^{(2)}=0 .
\end{gathered}
$$

The $2 \sqrt{7}$ denominator in Eq. (8) is introduced here for later convenience, in order to compare these expressions with those of Ref. [14]. The $\alpha_{2 \mu}$ variable behaves as the $\mathrm{O}(5) \supset \mathrm{O}(3)$ tensor $(\tau, L)=(1,2)$, while the second order term $[\alpha \times \alpha]_{\mu}^{(2)}$ is associated with the tensor $(2,2)[12]$. This means that they fulfill strict selection rules in the $\phi^{\tau \mu L M}\left(\gamma, \theta_{i}\right)$ space, $\Delta \tau= \pm 1$ for the former and $\Delta \tau=0$, \pm 2 for the latter, with the usual restrictions in angular momentum coupling $[2,12]$. Thus the first term leads to "allowed" transitions in first order and the second to nonzero quadrupole moments and to small values for "forbidden" ones, in the E(5) scheme of Ref. [5]. The actual values of these observables in $\mathrm{E}(5)$ depend on the simple $\beta$ dependence in the operators (8) and (9).

The results presented in [5] correspond to the energies (6) and transition operators (8) and (9) with $\chi=0$, i.e., for a $T^{(E 2)}$ of the form $T^{(E 2)}=t \alpha_{2 \mu}$, and the analysis is expected to apply in nuclei undergoing a transition between spherical and $\gamma$-unstable shapes. As explained above, the use of the second order form for $T^{(E 2)}$ of Eqs. (8) and (9) does not modify the $\Delta \tau= \pm 1$ transitions in Ref. [5] but gives rise to small values for the $\Delta \tau=0, \pm 2$ matrix elements. These results arise in the framework of the geometric model. Since we would compare the E(5) scheme with IBM calculations, however, we shall define an alternative $T^{(E 2)}$ operator that has a different $\beta$ dependence, consistent with the usual IBM quadrupole operator [14] as we explain below. In Refs. $[7,14]$ it has been shown that ${ }^{134} \mathrm{Ba}$ constitutes a good candidate for $\mathrm{E}(5)$ behavior. In [14] the IBM-consistent form for the $T^{(E 2)}$ operator was shown to lead to a closer agreement with experiment.

In what follows we consider a global IBM calculation for the $\mathrm{Ru}$ isotopes and compare the $\mathrm{E}(5)$ predictions (using the quadratic forms of both the geometric and IBM $T^{(E 2)}$ operators) with the transitional nucleus ${ }^{104} \mathrm{Ru}$.

\section{THE Ru ISOTOPES IN THE IBM}

The $\mathrm{Ru}$ isotopes display a transitional behavior from spherical [SU(5)] to $\gamma$-unstable [SO(6)] shapes [9]. As mentioned above, this is precisely the situation in which $\mathrm{E}(5)$ is expected to be relevant. A systematic study of the $\mathrm{Ru}$ isotopes was carried out by a simultaneous least-square fit to the energies of these nuclei in Ref. [9]. The converged Hamiltonian has the form

$$
H=\epsilon^{\prime} \hat{n}_{d}+\alpha \hat{N} \hat{n}_{d}+\beta C_{2}[\mathrm{SO}(6)]+\gamma C_{2}[\mathrm{SO}(5)]+\delta L^{2},
$$

with parameters (all in $\mathrm{keV}$ ) $\epsilon^{\prime}=887, \alpha=-53, \quad \beta$ $=-23.3, \gamma=30.8$, and $\delta=5.5$, while $C_{2}$ stands for the quadratic Casimir operator of the corresponding group and $N$ is the boson number. Here we prefer to use the equivalent Hamiltonian written in terms of a multipole expansion,

$$
H=\epsilon \hat{n}_{d}+\kappa_{0} P^{\dagger} P+\kappa_{1} L L+\kappa_{3} T_{3} T_{3}-\frac{\kappa_{0}}{4} N(N+4) .
$$

In this case the different operators are defined by

$$
\hat{n}_{d}=\sum_{\mu} d_{\mu}^{\dagger} d_{\mu}
$$




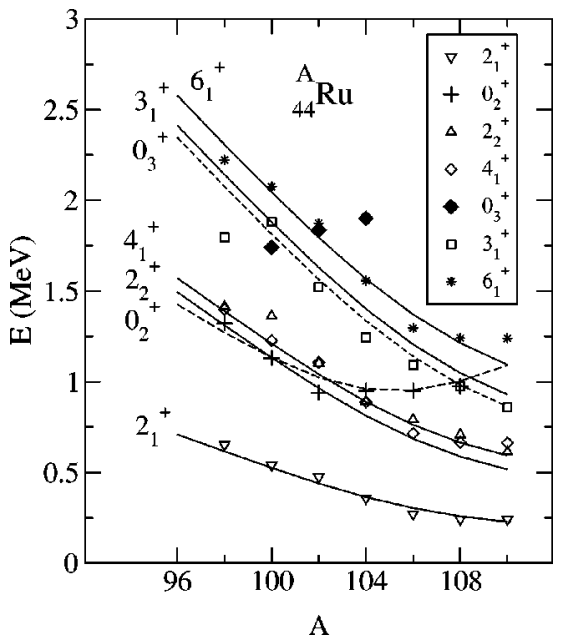

FIG. 1. Low-lying energy level systematics for the Ru isotopes ( $A=96-110$, corresponding to boson number $N=4-11$ ). Experimental and calculated results with Hamiltonian (11) with parameters given in the text are presented.

$$
\begin{gathered}
P^{\dagger}=\frac{1}{2}\left(d^{\dagger} d^{\dagger}-s^{\dagger} s^{\dagger}\right), \\
L=\sqrt{10}\left(d^{\dagger} \times d\right)^{(1)}, \\
T_{3}=\left(d^{\dagger} \times d\right)^{(3)} .
\end{gathered}
$$

With the appropriate translation of the parameters, the equivalent Hamiltonian to Eq. (10) is obtained with the values (all in keV) $\epsilon=887-53 N, \kappa_{0}=93.2, \kappa_{1}=11.66$, and $\kappa_{3}=61.6$. The parameters are kept fixed for all the isotopes studied in this work, the only variation in going from one isotope to the other is the change in $\epsilon$ induced by the variation of the boson number $N$. The boson number is obtained in this work by considering closed shells at 50 both for neutrons and protons. In Fig. 1 we show a comparison of the experimental and theoretical systematics of some low-lying levels in the $\mathrm{Ru}$ isotopes from $A=96$ to 110 , which correspond to boson number ranging from $N=4$ to 11 . In Fig. 2

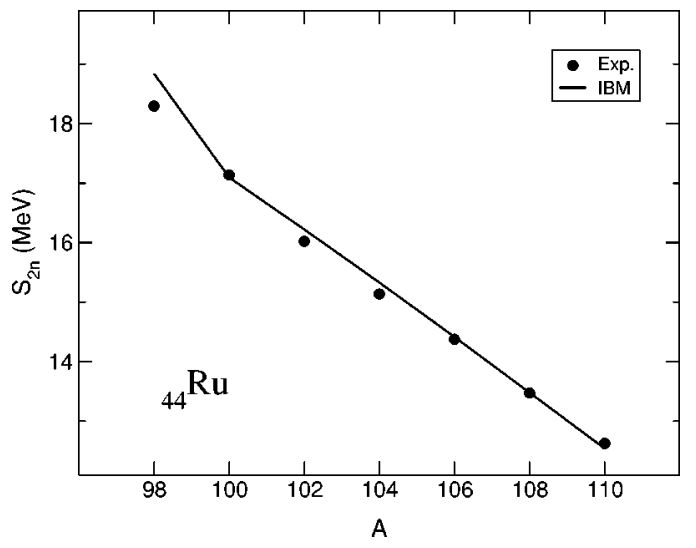

FIG. 2. Two-neutron separation energies in $\mathrm{Ru}$ isotopes. The IBM results correspond to the Hamiltonian (11) with the parameters given in the text. we show the results for two-neutron separation energies for this Hamiltonian. We see in Fig. 1 that Hamiltonian (10) and (11) leads to a good global description of the complete Ru isotope chain. We would point out three aspects of this figure. First, the systematics of the measured $0_{3}^{+}$is opposite to the expected one for a member of the three phonon multiplet. This seems to indicate that, as already proposed in Ref. [15], the $0^{+}$member of the three phonon multiplet is still missing experimentally. Second, the states $3_{1}^{+}$are systematically calculated too high in energy. This was proposed, first in Ref. [16] and then analyzed in detail in Ref. [15], as an indication of the presence of a mixed symmetry state. Finally, note the changing energies of the levels and particularly that of the $\mathrm{O}_{2}^{+}$state, suggesting a transitional behavior near ${ }^{104} \mathrm{Ru}$, although this is not reflected in the separation energies. This is known to be the case for a second order phase transition, as it is the transition from $\mathrm{U}(5)$ to $\mathrm{SO}(6)$.

In this paper we analyze the properties of the $\mathrm{Ru}$ isotopes in order to investigate the phase transition related to a change of shape. The global fit we are considering guarantees that the parameters are well determined. A geometrical interpretation of the abstract Hamiltonian (11) can be obtained by introducing coherent states [17-19] that allow to associate to it geometrical shapes in terms of the deformation variables $(\beta, \gamma)$. In this case the potential shapes will only depend on the value of $N$. The basic idea of this formalism is to consider that the pure quadrupole states are described by a boson condensate of the form

$$
|g ; N, \beta, \gamma\rangle=\frac{1}{\sqrt{N !}}\left(\Gamma_{g}^{\dagger}\right)^{N}|0\rangle,
$$

where the coherent boson is given by

$$
\Gamma_{g}^{\dagger}=\frac{1}{\sqrt{1+\beta^{2}}}\left[s^{\dagger}+\beta \cos \gamma d_{0}^{\dagger}+\frac{1}{\sqrt{2}} \beta \sin \gamma\left(d_{2}^{\dagger}+d_{-2}^{\dagger}\right)\right],
$$

which depends on the $\beta$ and $\gamma$ shape variables. The energy surface is then defined as

$$
E_{N}(\beta, \gamma)=\langle g ; N, \beta, \gamma|H| g ; N, \beta, \gamma\rangle
$$

where $H$ is given in our case by Eq. (11), which leads to potential energy surfaces that are $\gamma$ independent. In Fig. 3 these surfaces are plotted as a function of the deformation parameter $\beta$ for the different Ru isotopes. It is seen that for $N$ around 8 , which corresponds to ${ }^{104} \mathrm{Ru}$, a rather flat behavior of the energy surface as a function of $\beta$ is indeed obtained. This kind of potential is thus similar to the one corresponding to $\mathrm{E}(5)$ [5].

For a $\gamma$-independent Hamiltonian, the critical point $N_{c}$ can be defined as the value of $N$ for which the second derivative of the potential energy surface becomes null at $\beta$ $=0$. At values smaller than $N_{c}$ there is a spherical minimum, while for larger values the minimum is deformed. For the case of the Hamiltonian (11) we find 


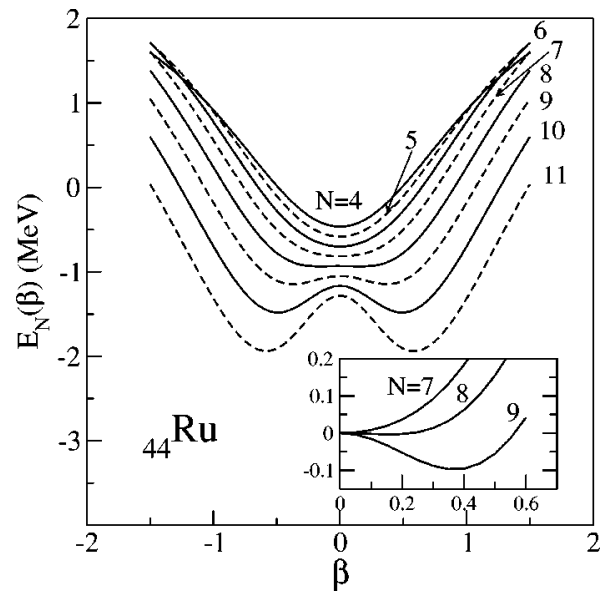

FIG. 3. Energy surfaces (18) for the Hamiltonian (11), with the parameters given in the text, as a function of the deformation parameter $\beta$ for the different $\mathrm{Ru}$ isotopes from boson number $N=4$ to $N=11$. In the inset the same is plotted in an expanded scale for the isotopes close to the critical point $N=7,8$, and 9 .

$$
N_{c}=\frac{5 \epsilon+5 \kappa_{0}+30 \kappa_{1}+7 \kappa_{3}}{5\left(\kappa_{0}+\alpha\right)},
$$

which for the parameters used in this work gives the value $N_{c}=7.77$, close to the value $N=8$ mentioned above. In the inset of Fig. 3 the potential energy surfaces are plotted for $N=7,8$, and 9 in an amplified scale, in order to show the transition from spherical to deformed shapes as a function of $N$. An appropriate constant factor was added to each curve in order to place them at zero energy for $\beta=0$. At this scale it is confirmed that $N=8$ is almost flat in $\beta$, as required by the $\mathrm{E}(5)$ prescription. Thus, ${ }^{104} \mathrm{Ru}$ is close to the critical point and should be a good candidate for testing the $\mathrm{E}(5)$ predictions. We stress that we have not adjusted or fine tuned the Hamiltonian parameters in any way, but have determined them from the global fit to the isotope series.

In addition to the energies, E2 transitions can be easily studied in the IBM. The E2 transition operator can be written as

$$
T_{\mu}^{(\mathrm{E} 2)}=q Q_{\mu}^{(s d)},
$$

where $q$ is a scale factor and $Q^{(s d)}$ is the general IBM quadrupole operator,

$$
Q_{\mu}^{(s d)}=\left(s^{\dagger} \widetilde{d}+d^{\dagger} s\right)_{\mu}^{(2)}+\chi\left(d^{\dagger} \widetilde{d}\right)_{\mu}^{(2)}
$$

The operators $\widetilde{d}_{m}=(-1)^{m} d_{-m}$ are introduced to behave as tensors with appropriate properties under spatial rotations. As discussed above, in Ref. [5] the simplest form of the E2 operator (8) and (9), corresponding to $\chi=0$ was used, while in Ref. [14] a form consistent with Eq. (21) was proposed for comparison with experimental data in ${ }^{134} \mathrm{Ba}$. By computing the expectation values of the $Q_{\mu}^{(s d)}$ operator in the coherent state (16), one finds the result for the intrinsic quadrupole operators $[14,20]$

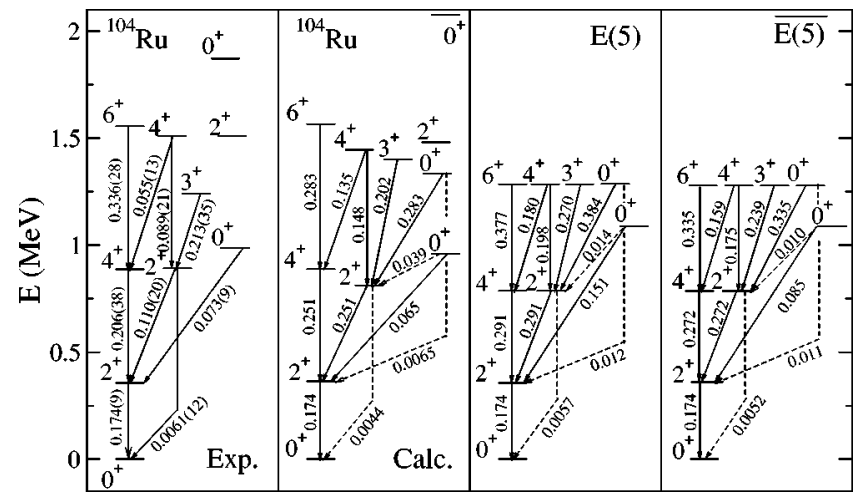

FIG. 4. Experimental data [energies and $\mathrm{B}(\mathrm{E} 2)$ values in $e^{2} \mathrm{~b}^{2}$ units] for ${ }^{104} \mathrm{Ru}$ [15] compared with the IBM calculation presented in this paper and with the results of the $\mathrm{E}(5)$ symmetry [5] with two elections for the $E 2$ transition operator. In the panel labeled $\mathrm{E}(5)$ the geometrical operators, Eqs. (8) and (9) have been used. In the panel labeled $\overline{\mathrm{E}(5)}$ the IBM operators, Eqs. (20) and (22), were used. In all cases, full lines indicate transitions allowed for $\chi=0$ and broken lines transitions only allowed if $\chi \neq 0$. The numbers given in this last case correspond to $\chi=1$. The scales for the energies and $B(E 2)$ 's are fixed by the experimental values of the excitation energy of the $2_{1}^{+}$and the $B\left(E 2 ; 2_{1}^{+} \rightarrow 0_{1}^{+}\right)$, respectively.

$$
\begin{gathered}
Q_{0}^{s d}(\beta, \gamma)=\frac{2 N}{1+\beta^{2}}\left[\beta \cos \gamma-\sqrt{\frac{1}{14}} \chi \beta^{2} \cos (2 \gamma)\right], \\
Q_{2}^{s d}(\beta, \gamma)=\frac{2 N}{1+\beta^{2}} \frac{1}{\sqrt{2}}\left[\beta \sin \gamma+\sqrt{\frac{1}{14}} \chi \beta^{2} \sin (2 \gamma)\right],
\end{gathered}
$$

to be compared with expressions (9). We note that the only difference is the common factor $2 N /\left(1+\beta^{2}\right)$. While the $N$ dependence has no consequence as can be incorporated into a renormalized value of the effective charge, the $\beta$-dependent denominator affects the magnitudes of the matrix elements. Even for $\chi=0$ in Eq. (22) the $\Delta \tau= \pm 1$ matrix elements are significantly affected. As shown in Ref. [14], by using the IBM form (22) in conjunction with the fivedimensional square-well potential wave functions (5), leads to a better agreement with the data in ${ }^{134} \mathrm{Ba}$. We shall see that the same is true for the transitional nucleus ${ }^{104} \mathrm{Ru}$. We show below the results of our calculations using the Hamiltonian (11) and quadrupole operator (21), when compared with the $\mathrm{E}(5)$ and modified-E(5) predictions using the quadrupole operators (22) with $\chi \neq 0$. We henceforth denote the latter by $\overline{\mathrm{E}(5)}$.

In Fig. 4 the known experimental data for the low-lying levels in ${ }^{104} \mathrm{Ru}$ are compared with the theoretical calculation and the predictions based on the potential (3). The calculated $B(E 2)$ values in the second panel are obtained with the IBM calculation presented in this section and Eqs. (20) and (21). Those in panels three and four are obtained for the potential (3) with two elections for the $E 2$ transition operator, Eqs. (8) and (9) in the panel labeled E(5) and Eqs. (20) and (22) in the panel labeled $\overline{\mathrm{E}(5)}$. In all cases, full lines indicate transitions allowed for $\chi=0(\Delta \tau= \pm 1)$ and broken lines transi- 

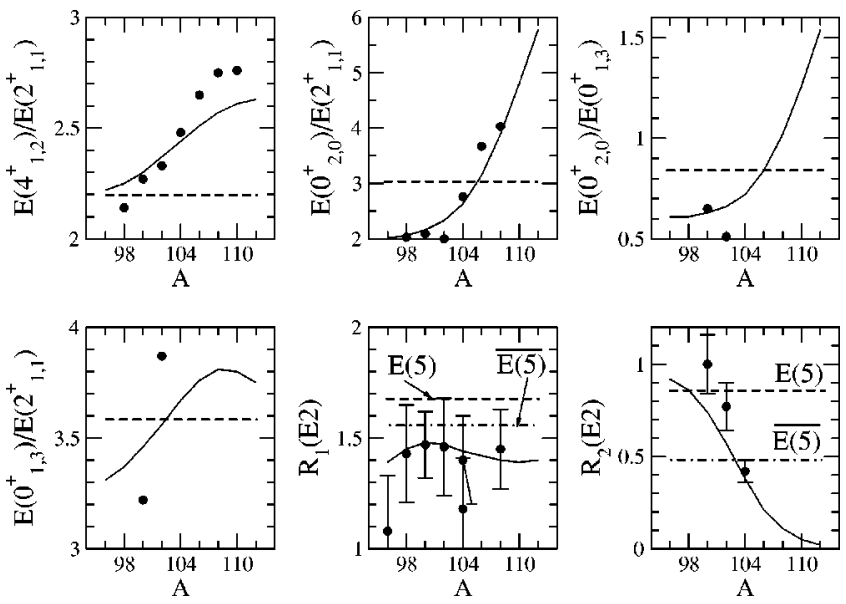

FIG. 5. Systematics of several calculated energy and $B(E 2)$ ratios as a function of $A$ for the $\mathrm{Ru}$ isotopes compared with the results of the $\mathrm{E}(5)$ symmetry (dashed lines). For $B(E 2)$ values also the values of the $\overline{\mathrm{E}(5)}$ symmetry with $\chi=1$ is shown (dotteddashed line). The IBM calculation (full line) was done with Hamiltonian (11) and the parameters given in the text. $R_{1}(E 2)$ $=B\left(E 2 ; 4_{1,2}^{+} \rightarrow 2_{1,1}^{+}\right) / B\left(E 2 ; 2_{1,1}^{+} \rightarrow 0_{1,0}^{+}\right) \quad$ and $\quad R_{2}(E 2)=B\left(E 2 ; 0_{2,0}^{+}\right.$ $\left.\rightarrow 2_{1,1}^{+}\right) / B\left(E 2 ; 2_{1,1}^{+} \rightarrow 0_{1,0}^{+}\right)$. The experimental data are taken from Refs. [15,21].

tions only allowed if $\chi \neq 0$. The numbers given in this last case correspond to $\chi=1$. In the IBM calculation (second panel) the boson effective charge is calculated to be $q$ $=0.106 e \mathrm{~b}$ which is very close to the value calculated in Ref. [16]. In the $\overline{\mathrm{E}(5)}$ case, with the IBM transition operators from Eqs. (20) and (22), the calculated effective charge is $q=0.136 e \mathrm{~b}$ for a value $\beta_{\omega}=1.0$ [which is the $\mathrm{SO}(6)$ value in the large $N$ limit] of the potential range (3).

In Fig. 5 we present the behavior of some energy and $B(E 2)$ ratios for the series of $\mathrm{Ru}$ isotopes and compare them with the critical symmetry. The available experimental data for the corresponding observables are shown, together with the $\mathrm{E}(5)$ and $\overline{\mathrm{E}(5)}$ values of Refs. [5,14]. The notation of the states is that of Ref. [5], $L_{\xi, \tau}^{\pi}$. We see that the region around

${ }^{104} \mathrm{Ru}$ is indeed surprisingly close to the $\overline{\mathrm{E}(5)}$ values. Although the experimental information on ${ }^{104} \mathrm{Ru}$ is not enough to make a definite statement about the validity of $\mathrm{E}(5)$ [or $\overline{\mathrm{E}(5)}]$ for this nucleus, we are able to remark the following:

(a) ${ }^{104} \mathrm{Ru}$ is closer to both predictions than any of the other isotopes, particularly if we make the comparison using the IBM form (22) for the $T^{(E 2)}$ operator $[\overline{\mathrm{E}(5)}]$.

(b) Since the IBM calculation seems to provide a very good general description of the whole isotope series, we feel reasonably confident about the predicted transition values in ${ }^{104} \mathrm{Ru}$ that are yet to be measured. These calculations closely resemble the values of $\overline{E(5)}$.

Two points should be commented combining Figs. 1, 4, and 5: (i) The energy ratio $4_{1}^{+} / 2_{1}^{+}$in ${ }^{104} \mathrm{Ru}$ shows an important anharmonicity and deviates from the $\mathrm{E}(5)$ value, (ii) it seems that the state $0^{+}$member of the three-phonon multiplet in ${ }^{104} \mathrm{Ru}$ (and probably in ${ }^{102} \mathrm{Ru}$ ) is experimentally missing and the one measured is a mixed-symmetry state (or has a large mixed-symmetry component). This could be also the case for the observed $3^{+}$in ${ }^{104} \mathrm{Ru}$ although the measured $B\left(E 2 ; 3_{1}^{+} \rightarrow 2_{2}^{+}\right)$fits nicely with the $\overline{\mathrm{E}(5)}$ value. Probably, as stated in Ref. [15], there are one full symmetry and one mixed-symmetry $3^{+}$states in the same energy region strongly mixed up. These points have to be investigated.

\section{CONCLUSIONS}

In this paper we have carried out a simultaneous fit to the $\mathrm{Ru}$ isotopes, using a global Hamiltonian with fixed parameters (except for the boson number $N$ ). These isotopes are well described by a transitional IBM Hamiltonian with a mixture of $\mathrm{U}(5)$ and $\mathrm{SO}(6)$ symmetries. The potentials arising from a coherent-state analysis indicate that ${ }^{104} \mathrm{Ru}$ is close to the critical point between spherical and $\gamma$-unstable structures. We have compared energy and $B(E 2)$ ratios for the $\mathrm{Ru}$ isotopes, and particularly ${ }^{104} \mathrm{Ru}$, with the recently proposed $\mathrm{E}$ (5) model of Ref. [5]. We find that the known experimental information on ${ }^{104} \mathrm{Ru}$ is well described by these critical symmetry, particularly if we use a modified form for the $T^{(E 2)}$ operator, consistent with the IBM quadrupole operator form (21) $[\overline{\mathrm{E}(5)}]$. Although the data are not sufficient to make a definite claim, the IBM predicted values and the $\overline{\mathrm{E}(5)}$ results are in excellent agreement.

Although these results are encouraging, we should point out that other examples of $\mathrm{E}(5)$ critical behavior should be sought, while a better understanding of the $\beta$ dependence of the quadrupole operator is required.

We are currently examining the $X(5)$ transitions using a similar approach. In summary, we believe that additional experimental and theoretical work is needed to assess the relevance of the $E(5)$ analytical description of critical behavior in nuclei.

\section{ACKNOWLEDGMENTS}

This work was supported in part by Conacyt under Project No. 32397-E and by the Spanish DGICYT under Project No. PB98-1111. We thank R. Bijker and J.E. García-Ramos for enlightening discussions.
[1] A. Bohr and B. Mottelson, Nuclear Structure (Benjamin, Reading, MA, 1975), Vol. II.

[2] F. Iachello and A. Arima, The Interacting Boson Model (Cambridge University Press, Cambridge, 1987).

[3] E. Lopez-Moreno and O. Castaños, Phys. Rev. C 54, 2374 (1996)
[4] R.F. Casten and D.D. Warner, Rev. Mod. Phys. 60, 389 (1988).

[5] F. Iachello, Phys. Rev. Lett. 85, 3580 (2000).

[6] F. Iachello, Phys. Rev. Lett. 87, 052502 (2001).

[7] R.F. Casten and N.V. Zamfir, Phys. Rev. Lett. 85, 3584 (2000).

[8] R.F. Casten and N.V. Zamfir, Phys. Rev. Lett. 87, 052503 (2001). 
[9] A. Frank, P. van Isacker, and D.D. Warner, Phys. Lett. B 197, 474 (1987).

[10] A. Frank, Phys. Rev. C 39, 652 (1989).

[11] J. M. Arias et al. (unpublished).

[12] E. Chacón, M. Moshinsky, and R.T. Sharp, J. Math. Phys. 17, 668 (1976).

[13] D. Bes, Nucl. Phys. 10, 373 (1959).

[14] J.M. Arias, Phys. Rev. C 63, 034308 (2001).

[15] A. Giannatiempo, A. Nannini, P. Sona, and D. Cutoiu, Phys. Rev. C 52, 2969 (1995).
[16] P. Van Isacker and G. Puddu, Nucl. Phys. A348, 125 (1980).

[17] J.N. Ginocchio and M.W. Kirson, Nucl. Phys. A350, 31 (1980).

[18] A.E.L. Dieperink, O. Scholten, and F. Iachello, Phys. Rev. Lett. 44, 1747 (1980).

[19] A. Bohr and B. Mottelson, Phys. Scr. 22, 468 (1980).

[20] C.E. Alonso, J.M. Arias, F. Iachello, and A. Vitturi, Nucl. Phys. A359, 59 (1992).

[21] S. Landsberger, R. Lecomte, P. Paradis, and S. Monaro, Phys. Rev. C 21, 588 (1980). 